\title{
Impact of front-of-pack labels on the perceived healthfulness of a sweetened fruit drink: a randomised experiment in five countries
}

\author{
Alejandra Jáuregui ${ }^{1, *} \odot$, Christine M White ${ }^{2}$, Lana Vanderlee ${ }^{3}$, Marissa G Hall ${ }^{4}$, \\ Alejandra Contreras-Manzano ${ }^{1} \odot$, Claudia Nieto', Gary Sacks ${ }^{5} \odot$, James F Thrasher ${ }^{6,7}$, \\ David Hammond ${ }^{2}$ and Simón Barquera ${ }^{1}$ \\ 'Nutrition and Health Research Center, National Institute of Public Health, Av. Universidad 655 Col. Santa María \\ Ahuacatitlán, CP, Cuernavaca, MR 62100, México: ${ }^{2}$ School of Public Health Sciences, University of Waterloo, \\ Waterloo, Ontario, Canada: ${ }^{3}$ École de Nutrition, Centre NUTRISS (nutrition, santé et société), Université Laval, \\ Québec, Québec, Canada: ${ }^{4}$ Department of Health Behavior, Gillings School of Global Public Health, Carolina \\ Population Center, Lineberger Comprehensive Cancer Center, University of North Carolina, Chapel Hill, NC, USA: \\ ${ }^{5}$ Global Obesity Centre, Deakin University, Burwood, Victoria, Australia: ${ }^{6}$ Arnold School of Public Health, University of \\ South Carolina, Columbia, SC, USA: ${ }^{7}$ Center for Population Health Research, National Institute of Public Health, \\ Cuernavaca, MR, México
}

Submitted 15 February 2021: Final revision received 13 0ctober 2021: Accepted 30 0ctober 2021: First published online 2 November 2021

\begin{abstract}
Objective: Front-of-pack (FOP) nutrition labelling is a globally recommended strategy to encourage healthier food choices. We evaluated the effect of FOP labels on the perceived healthfulness of a sweetened fruit drink in an international sample of adult consumers.

Design: Six-arm randomised controlled experiment to examine the impact of FOP labels (no label control, Guideline Daily Amounts (GDA), Multiple Traffic Lights, the Health Star Ratings (HSR), Health Warning Labels, and 'High-in' Warning Labels (HIWL)) on the perceived healthfulness of the drink. Linear regression models by country examined healthfulness perceptions on FOP nutrition labels, testing for interactions by demographic characteristics.

Setting: Online survey in 2018 among participants from Australia, Canada, Mexico, United Kingdom (UK) and United States.

Participants: Adults ( $\geq 18$ years, $n 22$ 140).

Results: Compared with control, HIWL had the greatest impact in lowering perceived healthfulness ( $\beta$ from -0.62 to -1.71 ) across all countries. The HIWL and the HSR had a similar effect in Australia. Other labels were effective in decreasing the perceived healthfulness of the drink within some countries only, but to a lower extent. The GDA did not reduce perceived healthfulness in most countries. In the UK, the effect of HIWL differed by age group, with greater impact among older participants ( $>40$ years). There were no other variations across key demographic characteristics.

Conclusions: HIWL, which communicates clear, non-quantitative messages about high levels of nutrients of concern, demonstrated the greatest efficacy to decrease the perceived healthfulness of a sweetened fruit drink across countries. This effect was similar across demographic characteristics.
\end{abstract}

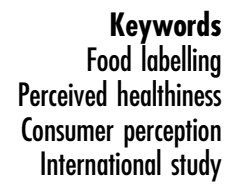

Keywords Perceived healthiness International study
Front-of-pack (FOP) nutrition labelling is a policy intervention to address the growing global burden of diet-related non-communicable diseases ${ }^{(1)}$. FOP labels aim to provide simplified or interpretative information on the nutritional quality or critical nutrient quantity (nutrients that may pose a substantial public health concern due to overconsumption, such as saturated fat, sugar and $\mathrm{Na})^{(2)}$ of a food product or about the health consequences of consuming nutrients or products, to help consumers make inferences about the healthfulness of the product and support more nutritious choices ${ }^{(3,4)}$. However, more research is needed to inform countries' decisions about which 
FOP system to use $\mathrm{e}^{(4)}$, and to assess potential differential effects among sub-groups ${ }^{(4,5)}$.

Various FOP labelling systems are implemented or being considered by governments globally ${ }^{(6)}$. Labelling systems can be classified as interpretive (i.e. providing nutrition information as guidance rather than specific facts) or reductive (i.e. showing information only, with no specific judgement, opinion or recommendation), as summary indicators (i.e. providing an overall qualification of the product healthfulness) or nutrient-specific systems (i.e. providing nutrition information for a set of nutrients $)^{(6)}$. Some of the most commonly employed systems include the Guideline Daily Amounts (GDA), Multiple Traffic Lights (MTL), Health Star Rating (HSR), 'High-in' Warning Labels (HIWL) and Health Warning Labels (HWL) (Fig. 1). GDA are a reductive approach with no interpretative information developed by the food industry, which provide information about the nutrient amounts within a food and its contribution to adult recommended daily intake. This labelling format is voluntarily implemented by the food industry in several countries, including Canada and the United States (US) and was mandatory in Mexico from 2014 to $2020^{(6)}$, when they were replaced by warning labels. MTL are interpretive nutrient-specific labels which provide similar information as GDA, but colour code each nutrient in order to communicate whether the product contains relatively low (green), average (yellow) or high (red) levels of critical nutrients. MTL have been implemented voluntarily in the United Kingdom (UK) since 2013, and approximately two-thirds of products in the UK carried the MTL in $2016^{(6,7)}$. MTL have been implemented similarly in other countries, including Ecuador ${ }^{(8)}$, Sri Lanka $^{(9)}$ and $\operatorname{Iran}^{(10)}$. The HSR, an interpretive summary indicator endorsed by the governments of Australia and New Zealand for voluntary implementation since 2014, synthesises positive and negative nutrient information into a single dimension of healthfulness, rating the overall nutritional quality of the product from 0.5 to 5 stars $^{(11)}$. In 2017 , the HSR system appeared on $28 \%$ of foods ${ }^{(6,12)}$. HIWL are interpretive nutrient-specific labels that show warning symbols (often octagonal) on food packages if energy and key nutrients (sugar, saturated fat and $\mathrm{Na}$ ) exceed established thresholds and were first introduced in Chile in $2015^{(6)}$. From an international regulatory and trade perspective, HIWL have been identified as a feasible mandatory system to implement ${ }^{(13)}$, and legislations for mandatory HIWL have been implemented in Israel, Uruguay, Peru and Mexico ${ }^{(6,14)}$, and proposed or approved in Brazil ${ }^{(15)}$, Argentina ${ }^{(16)}$ and Canada ${ }^{(17)}$. Lastly, an interpretive nutrient-specific text-only HWL for sugarsweetened beverage advertisements has been enacted in San Francisco, US but is being challenged in court and has also been proposed in seven US states ${ }^{(18)}$.

FOP labels are theorised to shape purchasing and consumption behaviours through several mechanisms. Once noticed by the consumer, FOP labels may change the motivation to consume food products by modifying the way in which they are perceived ${ }^{(19)}$. For example, highlighting the high content of nutrients of public health concern may decrease perceived healthfulness of a product previously misperceived as healthy (e.g. sweetened yogurt or sugary fruit drinks). Indeed real-life experiments suggest that changes in the perceived healthfulness of food products may influence consumption. ${ }^{(20,21)}$ Studies examining the effects of FOP labels on perceived healthfulness suggest that nutrient-specific labels (e.g. MTL, HIWL) and the HSR may be more effective in leading to lower ratings of unhealthy foods compared with GDA ${ }^{(5,22-24)}$. A meta-analysis of experimental studies found that sugar-sweetened beverage warnings (including both HIWL and HWL) successfully lowered healthfulness perceptions compared with control conditions ${ }^{(25)}$. Finally, a recent scoping review of experimental studies of HIWL reported that these labels led to lower perceived healthfulness of products compared
1)

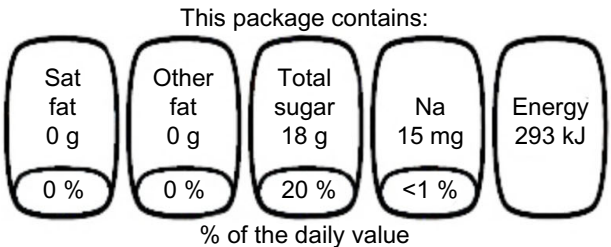

3)

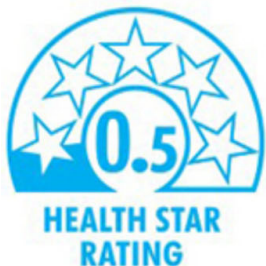

4)

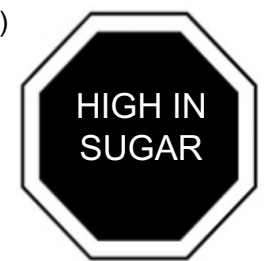

2)

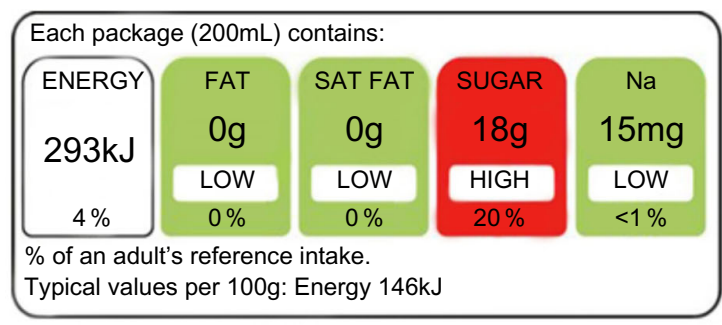

5)

WARNING: Drinking beverages with added sugar(s)

contributes to obesity, diabetes, and tooth decay.

Department of Health

Fig. 1 (colour online) Front-of-pack labels shown on product during experiment. (1) Guideline Daily Amounts (GDA), (2) Multiple Traffic Lights (MTL), (3) Health Star Rating (HSR), (4) 'High-in' Warning Labels (HIWL) and (5) Health Warning Labels (HWL) 
with control conditions or other labelling formats (i.e. GDA) ${ }^{(26)}$.

However, a key question is whether FOP label effects are generalisable across countries. Previous international studies exploring country differences on labelling outcomes (e.g. perceived product healthfulness, label perceptions) have found inconclusive results, with some reporting differences across countries ${ }^{(27-29)}$, whereas others have not $^{(30-32)}$. To date, most of these international studies have been conducted in Europe ${ }^{(27,29,31)}$, with less representation of other regions in the world ${ }^{(28,33)}$. Additionally, a call has been made to focus research on the reach of FOP labels' effects across sub-groups of consumers such as those with varying levels of nutrition knowledge, or among lowincome populations ${ }^{(5,26)}$.

The objective of this study was to test the effect of different kinds of FOP labels (GDA, MTL, HSR, HWL and HIWL) on the perceived product healthfulness in an international sample of adult consumers, including evaluation of differences by socio-demographic characteristics and country.

\section{Methods}

\section{Study design and recruitment}

A six-arm, unblinded online randomised experiment was conducted as part of the broader 2018 International Food Policy Study (IFPS), a cross-sectional survey of adults aged $\geq 18$ years ( $n 22$ 824) from Australia, Canada, Mexico, the UK and the US, who completed an online survey in 2018. The IFPS assesses seven primary policy domains including price/taxation, food packaging and labelling, retail food policies, food marketing, nutritional labelling in restaurants, nutrition information and education, and food guide/dietary recommendations. For the present study, we analysed responses to one single question regarding the perceived healthfulness of a fruit drink labelled with differing FOP labels. The countries represent different policy approaches to FOP labels, as outlined above.

Approximately $2.9 \%$ of participants ( $n$ 684) were excluded due to missing data in the outcome ( $n$ 634) or a technical glitch in the survey platform making participants view all experimental conditions on the screen $(n 50)$, leaving 22140 participants for analyses (Australia = 3964; Canada $=4311 ;$ Mexico $=4057 ; \quad$ UK $=5290 ; \quad$ US $=4518$ ). Small differences between included and excluded participants were observed $(P<0 \cdot 01)$ (online supplementary material, Supplemental Table 2). Missing data in the outcome across label conditions ranged from 1.8 to $3.7 \%$ $(P<0.001)$ (online supplementary material, Supplemental Table 3).

Participants were recruited through the Nielsen Consumer Insights Global Panel and their partners' panels using both probability and non-probability sampling methods. Random samples were drawn from online panels in each country, stratified by age and sex proportional to the general population in each country. Respondents provided consent prior to completing the survey and received remuneration in accordance with their panel's usual incentive structure (e.g. points-based or monetary rewards). Surveys were conducted in English in Australia and the UK; Spanish in Mexico; English or French in Canada; and English or Spanish in the US.

\section{Participants' allocation and intervention}

Using a central computer system, participants were randomly assigned to view on screen one of six images $(6.5$ $\mathrm{cm} \times 13 \mathrm{~cm}$ ) of a sweetened fruit drink with differing labelling: no label (control), GDA, MTL, HSR, HWL or HIWL (Fig. 1). These labelling systems were selected as they are either implemented or being considered as a policy option in the five IFPS countries. Researchers were blinded to the assigned intervention, but blinding of participants was not possible given the nature of the intervention.

FOP labels were displayed in the upper right corner of the front of the pack (Fig. 2 and online supplementary material, Supplemental Fig. 1). A sweetened fruit drink was utilised as the test product because processed fruit drinks are considered to be sugar-sweetened beverages and are commonly misperceived as healthy ${ }^{(34,35)}$, despite their high added sugar content and high contribution to energy intake ${ }^{(36)}$. The sweetened fruit drink box image was modelled after a popular drink package to appear authentic, but digitally altered to display fictitious brand names. Package text language and units of measures were altered to match typical product packaging in each country.

\section{Nutritional criteria for labelling systems}

Online supplementary material, Supplemental Table 1 shows the nutrition information used in the development of the FOP labels for the sweetened fruit drink. The nutrient content in the MTL condition was classified according to criteria set out by EU Regulation No. 1169/2011 (e.g. sugar content $>13.5 \mathrm{~g} /$ portion coded red with 'high' text ${ }^{(37)}$. The online HSR Calculator was utilised to calculate an HSR of 0.5 stars $^{(38)}$. The 'High in Sugar' warning label was applied based on a cut-off of $18 \mathrm{~g}$ of sugar per serving size ${ }^{(39)}$ or $5 \mathrm{~g} /$ $100 \mathrm{ml}$, as per criteria used in Chile ${ }^{(40)}$.

\section{Outcome}

Participants were asked 'In your opinion, is this product...' with nine response options: (1) very unhealthy, (2) unhealthy, (3) a little unhealthy, (4) neither unhealthy nor healthy,(5) a little healthy, (6) healthy, (7) very healthy, (8) don't know and (9) refuse to answer. Those answering options (8) or (9) ( $n$ 634, 2.9\%) were considered as missing and excluded from analyses. 
Front-of-pack labels and perceived healthfulness

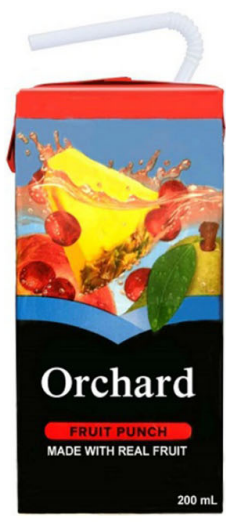

No label control

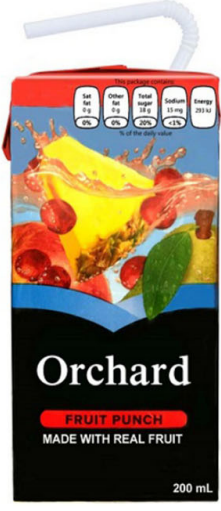

Guideline Daily Amounts

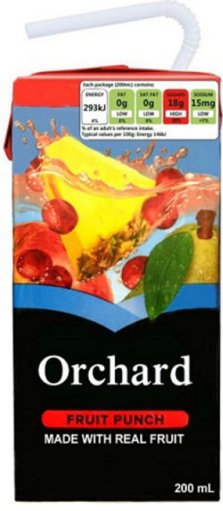

Multiple Traffic Lights

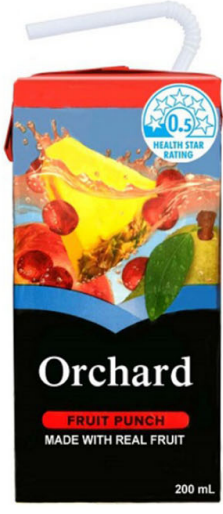

Health Star Rating

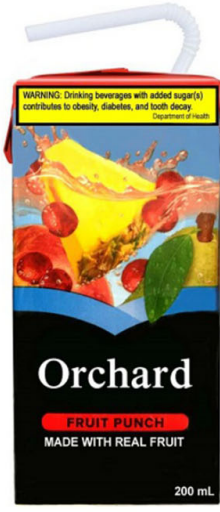

Health Warning

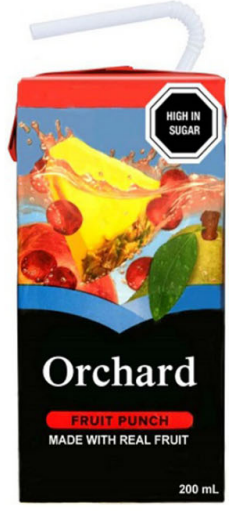

Nutrient-based Warning Labels

Fig. 2 (colour online) Images with front-of-package labels displayed on screen during experimental task. Note: each participant was only shown one image, corresponding to their assigned condition. Images above were shown in Australia surveys; product and labels varied slightly by country (see online supplementary material, Supplemental Fig. 1)

\section{Covariates}

Demographic information was assessed using survey measures $^{(41)}$ from population-level surveys within each country ${ }^{(42-46)}$. Variables were recoded and harmonised for comparison across countries and included gender, age group, education, ethnicity, income adequacy ${ }^{(47)}$, selfreported nutrition knowledge, household responsibility for food shopping, frequency of using a nutrition facts table and self-reported BMI (see Table 1). Further details on the IFPS are available elsewhere ${ }^{(48)}$.

\section{Statistical analysis}

The IFPS study sample size was powered to examine differences in nutritional outcomes between countries over time and not for each task within the survey. Post-hoc analyses indicated that with a sample size of 650 participants in each labelling condition per country and a standard deviation of 1.5 , this study had an estimated $85 \%$ power to detect a 0.25 mean difference on the 7 -point Likert scale. We tested the success of randomisation of covariates by comparing variables between experimental groups using $\chi^{2}$ tests.

Preliminary analyses indicated differences in label effects across countries (overall interaction effect: $\left.X^{2}=41.66, P=0.003\right)$; thus, separate country models were estimated. Linear regression modelling was used to evaluate the effect of the labels on perceived healthfulness. Comparisons among label groups were made using Wald tests after running linear regression models.

We tested for possible interactions between label condition and demographic characteristics (i.e. gender, age group, income adequacy, education, nutrition knowledge, food shopping in the household, frequency of using the nutrition facts table or BMI category). For this purpose, multiplicative interactions between each demographic variable and label condition were introduced in individual country models, but only significant interactions $(P<0.01)$ were retained. In cases where demographic $\times$ label interactions were significant, associations within the demographic variables were presented, stratified by country.

Additional sensitivity analyses were performed to check the robustness of the results. Participants considering the food product as very healthy (7), healthy (6) or a little healthy (5) were classified as perceiving the product as 'healthy'; those choosing options (4), (3), (2) or (1) were classified as perceiving the product as 'not healthy'. We regressed this binary outcome on the experimental group.

To account for the use of several models and multiple comparisons within each, significance was set at $P<0.01$ for regression models and test comparisons. All analyses were weighted with post-stratification sample weights constructed using a raking algorithm with population estimates from the census in each country based on age group, sex, region, ethnicity (except in Canada) and education (except in Mexico). Data analysis was performed using STATA 14.

\section{Results}

A total of 22140 participants were analysed (control =3612, $\mathrm{GDA}=3647, \quad \mathrm{MTL}=3711, \quad \mathrm{HSR}=3735$, HIWL $=3699$, HWL $=3736$ ). No differences were observed between experimental conditions in characteristics (Table 1). Participants were evenly distributed between conditions by country, gender, age group and education level. Most (70-80\%) belonged to a majority ethnic group and were responsible for food shopping in their household, with slightly more females than males.

Stratified models showed that HIWL were the most effective label in reducing the perceived healthfulness of the fruit drink compared with the control group in all countries (range of $\beta:-1.20$ in the UK to -0.62 in Canada), as 
Table 1 Demographic characteristics in the total sample and by experimental condition (weighted)

\begin{tabular}{|c|c|c|c|c|c|c|c|c|c|c|c|c|c|c|c|}
\hline & \multicolumn{2}{|c|}{ Total $(n 22$ 140) } & \multicolumn{2}{|c|}{$\begin{array}{l}\text { Control (no label) } \\
\quad(n 3612)\end{array}$} & \multicolumn{2}{|c|}{$\begin{array}{l}\text { Guideline Daily } \\
\text { Amounts (GDA) } \\
(n 3647)\end{array}$} & \multicolumn{2}{|c|}{$\begin{array}{l}\text { Multiple Traffic } \\
\text { Lights (MTL) } \\
(n 3711)\end{array}$} & \multicolumn{2}{|c|}{$\begin{array}{l}\text { Health Star } \\
\text { Rating (HSR) } \\
\text { (n 3735) }\end{array}$} & \multicolumn{2}{|c|}{$\begin{array}{l}\text { Health Warning } \\
\text { Label (HWL) } \\
(\text { ( } 3736)\end{array}$} & \multicolumn{2}{|c|}{$\begin{array}{l}\text { ‘High-in’ Warning } \\
\text { Label (HIWL) } \\
\text { (n 3699) }\end{array}$} & \multirow[b]{2}{*}{$P^{*}$} \\
\hline & $\%$ & $99 \% \mathrm{Cl}$ & $\%$ & $99 \% \mathrm{Cl}$ & $\%$ & $99 \% \mathrm{Cl}$ & $\%$ & $99 \% \mathrm{Cl}$ & $\%$ & $99 \% \mathrm{Cl}$ & $\%$ & $99 \% \mathrm{Cl}$ & $\%$ & $99 \% \mathrm{Cl}$ & \\
\hline \multicolumn{16}{|l|}{ Country } \\
\hline Australia & $17 \cdot 9$ & $17 \cdot 1,18 \cdot 7$ & $18 \cdot 0$ & $16 \cdot 2,20 \cdot 0$ & 18.5 & $16 \cdot 7,20 \cdot 5$ & $18 \cdot 0$ & $16 \cdot 2,20 \cdot 0$ & $18 \cdot 5$ & $16 \cdot 7,20 \cdot 5$ & $17 \cdot 3$ & $15 \cdot 5,19 \cdot 3$ & $17 \cdot 2$ & $15 \cdot 4,19 \cdot 1$ & 0.687 \\
\hline Canada & 19.5 & $18 \cdot 7,20 \cdot 3$ & $19 \cdot 2$ & $17 \cdot 3,21 \cdot 3$ & $19 \cdot 6$ & $17 \cdot 6,21 \cdot 7$ & $19 \cdot 2$ & $17 \cdot 3,21 \cdot 3$ & 19.5 & $17 \cdot 5,21 \cdot 5$ & $19 \cdot 9$ & $17 \cdot 9,22 \cdot 0$ & 19.5 & $17 \cdot 5,21 \cdot 6$ & \\
\hline Mexico & $18 \cdot 3$ & $17 \cdot 5,19 \cdot 2$ & $16 \cdot 8$ & $15 \cdot 0,18 \cdot 8$ & $18 \cdot 2$ & $16 \cdot 3,20 \cdot 3$ & $18 \cdot 9$ & $17 \cdot 0,21 \cdot 0$ & $18 \cdot 2$ & $16 \cdot 3,20 \cdot 2$ & $19 \cdot 0$ & $17 \cdot 1,21 \cdot 1$ & $18 \cdot 8$ & $16 \cdot 9,20 \cdot 9$ & \\
\hline United Kingdom & 23.9 & $23 \cdot 0,24 \cdot 8$ & 24.9 & $22 \cdot 7,27 \cdot 3$ & 23.5 & $21 \cdot 4,25 \cdot 7$ & $24 \cdot 3$ & $22 \cdot 2,26 \cdot 5$ & $23 \cdot 0$ & $20 \cdot 9,25 \cdot 2$ & $23 \cdot 4$ & $21 \cdot 3,25 \cdot 6$ & $24 \cdot 3$ & $22 \cdot 2,26 \cdot 4$ & \\
\hline United States & 20.4 & $19 \cdot 6,21 \cdot 3$ & $21 \cdot 0$ & $18 \cdot 9,23 \cdot 3$ & $20 \cdot 2$ & $18 \cdot 3,22 \cdot 3$ & $19 \cdot 6$ & $17 \cdot 7,21 \cdot 8$ & $20 \cdot 8$ & $18 \cdot 8,23 \cdot 0$ & $20 \cdot 4$ & $18 \cdot 3,22 \cdot 5$ & $20 \cdot 3$ & $18 \cdot 3,22 \cdot 4$ & \\
\hline \multicolumn{16}{|l|}{ Gender } \\
\hline Male & 48.5 & $47 \cdot 5,49 \cdot 6$ & $48 \cdot 6$ & $46 \cdot 0,51 \cdot 1$ & $48 \cdot 2$ & $45 \cdot 6,50 \cdot 8$ & $49 \cdot 2$ & $46 \cdot 7,51 \cdot 8$ & $49 \cdot 0$ & $46 \cdot 5,51 \cdot 5$ & $49 \cdot 4$ & $46 \cdot 9,52 \cdot 0$ & $46 \cdot 7$ & $44 \cdot 2,49 \cdot 2$ & 0.389 \\
\hline Female & 51.5 & $50 \cdot 4,52 \cdot 5$ & 51.4 & $48.9,54.0$ & $51 \cdot 8$ & $49 \cdot 2,54 \cdot 3$ & $50 \cdot 8$ & $48 \cdot 2,53 \cdot 3$ & $51 \cdot 0$ & $48 \cdot 4,53.5$ & $50 \cdot 6$ & $48 \cdot 0,53 \cdot 1$ & $53 \cdot 3$ & $50 \cdot 8,55 \cdot 8$ & \\
\hline \multicolumn{16}{|l|}{ Ethnicity ${ }^{\dagger}$} \\
\hline Majority group & $80 \cdot 0$ & $79 \cdot 0,80 \cdot 8$ & $79 \cdot 7$ & $77 \cdot 3,81 \cdot 9$ & 79.5 & $77 \cdot 2,81 \cdot 7$ & $79 \cdot 9$ & $77 \cdot 5,82 \cdot 0$ & $79 \cdot 4$ & $77 \cdot 1,81 \cdot 5$ & $81 \cdot 0$ & $78 \cdot 8,83 \cdot 1$ & $80 \cdot 2$ & $77 \cdot 9,82 \cdot 3$ & 0.975 \\
\hline Minority group & $20 \cdot 0$ & $19 \cdot 2,21 \cdot 0$ & $20 \cdot 3$ & $18 \cdot 1,22 \cdot 7$ & 20.5 & $18 \cdot 3,22 \cdot 8$ & $20 \cdot 1$ & $18 \cdot 0,22 \cdot 4$ & $20 \cdot 6$ & $18 \cdot 5,22.9$ & $19 \cdot 0$ & $16 \cdot 9,21 \cdot 2$ & $19 \cdot 8$ & $17 \cdot 7,22 \cdot 1$ & \\
\hline \multicolumn{16}{|l|}{ Age group } \\
\hline $18-29$ & 22.5 & $21 \cdot 6,23 \cdot 4$ & 22.5 & $20 \cdot 3,24 \cdot 7$ & $21 \cdot 3$ & $19 \cdot 2,23 \cdot 5$ & $23 \cdot 4$ & $21 \cdot 5,25 \cdot 9$ & $22 \cdot 6$ & $20 \cdot 5,24 \cdot 7$ & $21 \cdot 2$ & $20 \cdot 1,224 \cdot 3$ & $22 \cdot 9$ & $20 \cdot 8,25 \cdot 1$ & $0 \cdot 201$ \\
\hline $30-39$ & $18 \cdot 3$ & $17 \cdot 5,19 \cdot 1$ & 17.5 & $15 \cdot 8,19 \cdot 5$ & $18 \cdot 6$ & $16 \cdot 7,20 \cdot 6$ & $18 \cdot 2$ & $16 \cdot 4,20 \cdot 1$ & $17 \cdot 7$ & $15 \cdot 8,19 \cdot 6$ & $19 \cdot 3$ & $17 \cdot 4,21 \cdot 3$ & $18 \cdot 3$ & $16 \cdot 5,20 \cdot 3$ & \\
\hline $40-49$ & $16 \cdot 2$ & $15 \cdot 5,17 \cdot 0$ & $16 \cdot 1$ & $14 \cdot 3,18 \cdot 1$ & $16 \cdot 8$ & $15 \cdot 0,18 \cdot 9$ & $16 \cdot 1$ & $14 \cdot 4,18 \cdot 1$ & $16 \cdot 9$ & $15 \cdot 1,19 \cdot 0$ & $15 \cdot 9$ & $14 \cdot 1,17 \cdot 9$ & $15 \cdot 4$ & $13 \cdot 7,17 \cdot 3$ & \\
\hline $50-59$ & $18 \cdot 0$ & $17 \cdot 2,18 \cdot 9$ & 18.5 & $16 \cdot 5,20 \cdot 8$ & $17 \cdot 8$ & $15 \cdot 9,20 \cdot 1$ & $17 \cdot 9$ & $16 \cdot 0,20 \cdot 1$ & $18 \cdot 9$ & $16 \cdot 9,21 \cdot 0$ & $17 \cdot 8$ & $15 \cdot 8,19 \cdot 9$ & $17 \cdot 4$ & $15 \cdot 4,19 \cdot 5$ & \\
\hline $60-69$ & $16 \cdot 2$ & $15 \cdot 4,16 \cdot 9$ & $17 \cdot 1$ & $15 \cdot 2,18 \cdot 9$ & $16 \cdot 7$ & $14 \cdot 7,18 \cdot 3$ & 14.7 & $13 \cdot 0,16 \cdot 4$ & $15 \cdot 4$ & $13 \cdot 5,17 \cdot 0$ & $16 \cdot 4$ & $14 \cdot 6,18 \cdot 2$ & $17 \cdot 4$ & $15 \cdot 5,19 \cdot 3$ & \\
\hline 70 and over & $8 \cdot 8$ & $8 \cdot 3,9 \cdot 4$ & 8.4 & $7 \cdot 1,9 \cdot 8$ & $9 \cdot 0$ & $7 \cdot 7,10 \cdot 6$ & 9.5 & $8 \cdot 1,11 \cdot 1$ & $8 \cdot 7$ & $7 \cdot 4,10 \cdot 3$ & $8 \cdot 6$ & $7 \cdot 3,10 \cdot 0$ & $9 \cdot 0$ & $7 \cdot 4,10 \cdot 2$ & \\
\hline \multicolumn{16}{|l|}{ Education level ${ }^{\ddagger}$} \\
\hline Low & $42 \cdot 8$ & $41 \cdot 8,43 \cdot 9$ & $43 \cdot 4$ & $40 \cdot 8,46 \cdot 1$ & $42 \cdot 3$ & $39 \cdot 7,45 \cdot 0$ & 41.9 & $39 \cdot 3,44 \cdot 6$ & $44 \cdot 3$ & $41 \cdot 7,46 \cdot 9$ & 42.5 & $39 \cdot 9,45 \cdot 1$ & $42 \cdot 5$ & $39 \cdot 9,45 \cdot 1$ & 0.169 \\
\hline Medium & $22 \cdot 2$ & $21 \cdot 4,23 \cdot 0$ & $22 \cdot 1$ & $20 \cdot 3,24 \cdot 1$ & 23.4 & $21 \cdot 5,25 \cdot 4$ & $23 \cdot 3$ & $21 \cdot 4,25 \cdot 3$ & 21.9 & $20 \cdot 0,23 \cdot 8$ & $21 \cdot 7$ & $19 \cdot 9,23 \cdot 6$ & $20 \cdot 9$ & $19 \cdot 1,22 \cdot 8$ & \\
\hline High & $35 \cdot 0$ & $34 \cdot 0,35 \cdot 9$ & 34.4 & $32 \cdot 2,36 \cdot 7$ & $34 \cdot 3$ & $32 \cdot 1,36 \cdot 6$ & $34 \cdot 8$ & $32 \cdot 6,37 \cdot 1$ & 33.9 & $31 \cdot 7,36 \cdot 1$ & $35 \cdot 8$ & $33 \cdot 5,38 \cdot 1$ & $36 \cdot 6$ & $34.3,38.9$ & \\
\hline \multicolumn{16}{|l|}{ Income adequacy§ } \\
\hline Very difficult/difficult & $30 \cdot 7$ & $29 \cdot 7,31 \cdot 7$ & $29 \cdot 7$ & $27 \cdot 3,32 \cdot 2$ & $30 \cdot 9$ & $28 \cdot 6,33 \cdot 3$ & $30 \cdot 7$ & $28 \cdot 3,33 \cdot 2$ & $30 \cdot 6$ & $28 \cdot 2,33 \cdot 0$ & $32 \cdot 3$ & $29 \cdot 9,34 \cdot 8$ & $29 \cdot 8$ & $27 \cdot 6,32 \cdot 3$ & 0.544 \\
\hline Neither easy nor difficult & $36 \cdot 7$ & $35 \cdot 7,37 \cdot 7$ & $37 \cdot 6$ & $35 \cdot 1,40 \cdot 1$ & $36 \cdot 2$ & $32 \cdot 7,38 \cdot 7$ & $37 \cdot 2$ & $34 \cdot 7,39 \cdot 7$ & $36 \cdot 5$ & $34 \cdot 1,39 \cdot 0$ & $35 \cdot 5$ & $33 \cdot 1,38 \cdot 0$ & 37.4 & $35 \cdot 0,39 \cdot 9$ & \\
\hline Easy/very easy & $32 \cdot 6$ & $31.7,33.5$ & $32 \cdot 7$ & $30.4,35.1$ & $32 \cdot 9$ & $30 \cdot 7,35 \cdot 2$ & $32 \cdot 1$ & $29 \cdot 9,34.4$ & 32.9 & $30 \cdot 6,35 \cdot 2$ & $32 \cdot 2$ & $30.0,34.5$ & $32 \cdot 7$ & $30 \cdot 4,35 \cdot 1$ & \\
\hline \multicolumn{16}{|l|}{ Nutrition knowledge\| } \\
\hline Not at all or a little knowledgeable & $37 \cdot 6$ & $36 \cdot 5,38 \cdot 6$ & $38 \cdot 0$ & $35 \cdot 5,40 \cdot 6$ & $36 \cdot 5$ & $34 \cdot 1,39 \cdot 0$ & $38 \cdot 0$ & $35 \cdot 5,40 \cdot 5$ & $36 \cdot 3$ & $33 \cdot 9,38.8$ & $39 \cdot 1$ & $36 \cdot 7,41 \cdot 7$ & $37 \cdot 8$ & $35 \cdot 3,40 \cdot 3$ & 0.030 \\
\hline Somewhat knowledgeable & $43 \cdot 1$ & $42 \cdot 1,44 \cdot 1$ & 42.9 & $40.5,45 \cdot 5$ & 43.5 & $41 \cdot 0,46 \cdot 0$ & $42 \cdot 3$ & $39 \cdot 8,44 \cdot 8$ & 44.4 & $41.9,47.0$ & $41 \cdot 2$ & $38 \cdot 7,43 \cdot 7$ & 44.0 & $41 \cdot 5,46 \cdot 6$ & \\
\hline Very or extremely knowledgeable & $19 \cdot 3$ & $18 \cdot 5,20 \cdot 1$ & $19 \cdot 0$ & $17 \cdot 2,21 \cdot 1$ & $20 \cdot 0$ & $18 \cdot 1,22 \cdot 0$ & $19 \cdot 7$ & $18 \cdot 1,22 \cdot 0$ & $19 \cdot 3$ & $17 \cdot 8,21 \cdot 8$ & $19 \cdot 6$ & $17 \cdot 8,21 \cdot 6$ & $18 \cdot 2$ & $16 \cdot 8,20 \cdot 2$ & \\
\hline \multicolumn{16}{|l|}{ Food shopping in your household" } \\
\hline Yes & $73 \cdot 1$ & $72 \cdot 1,74 \cdot 0$ & 73.5 & $71 \cdot 2,75 \cdot 7$ & $72 \cdot 5$ & $70 \cdot 1,74 \cdot 7$ & $72 \cdot 0$ & $69 \cdot 6,74.2$ & $72 \cdot 1$ & $69 \cdot 8,74 \cdot 3$ & $74 \cdot 1$ & $71 \cdot 9,76 \cdot 3$ & 74.4 & $72 \cdot 2,76 \cdot 6$ & 0.748 \\
\hline No & $6 \cdot 0$ & $5 \cdot 5,6 \cdot 6$ & $5 \cdot 6$ & $4 \cdot 4,7 \cdot 0$ & $5 \cdot 8$ & $4 \cdot 7,7 \cdot 2$ & $6 \cdot 1$ & $5 \cdot 0,7 \cdot 5$ & 6.5 & $5 \cdot 3,8 \cdot 0$ & $6 \cdot 4$ & $5 \cdot 2,7 \cdot 9$ & $5 \cdot 6$ & $4.5,6.9$ & \\
\hline Share & $20 \cdot 9$ & $20 \cdot 0,21 \cdot 7$ & $20 \cdot 9$ & $18 \cdot 9,23 \cdot 0$ & $21 \cdot 7$ & $19 \cdot 7,23 \cdot 8$ & 21.9 & $19 \cdot 8,24 \cdot 0$ & 21.4 & $19 \cdot 4,23.5$ & 19.5 & $17 \cdot 6,21 \cdot 5$ & $20 \cdot 0$ & $18 \cdot 1,22 \cdot 1$ & \\
\hline \multicolumn{16}{|c|}{ Frequency of using nutrition information ${ }^{\star \star}$} \\
\hline Never/rarely & $24 \cdot 8$ & $23 \cdot 9,25 \cdot 8$ & $24 \cdot 1$ & $21 \cdot 9,26 \cdot 4$ & $25 \cdot 0$ & $22 \cdot 8,27 \cdot 4$ & $24 \cdot 7$ & $22 \cdot 5,26 \cdot 9$ & 24.4 & $22 \cdot 3,26 \cdot 8$ & $25 \cdot 5$ & $23 \cdot 3,27 \cdot 8$ & $25 \cdot 3$ & $23 \cdot 1,27 \cdot 7$ & 0.729 \\
\hline Sometimes & $31 \cdot 8$ & $30 \cdot 9,32 \cdot 8$ & $32 \cdot 3$ & $29 \cdot 9,34.7$ & $32 \cdot 3$ & $29.9,34.7$ & 31.5 & $29 \cdot 2,33.9$ & $31 \cdot 2$ & $29 \cdot 2,33 \cdot 9$ & 31.5 & $29 \cdot 2,33 \cdot 9$ & $32 \cdot 3$ & $29 \cdot 9,34 \cdot 7$ & \\
\hline Often/all the time & $43 \cdot 3$ & $42 \cdot 3,44 \cdot 4$ & $43 \cdot 7$ & $41 \cdot 1,46 \cdot 2$ & $42 \cdot 7$ & $40 \cdot 2,45 \cdot 2$ & $43 \cdot 8$ & $41 \cdot 3,46 \cdot 4$ & $44 \cdot 4$ & $41 \cdot 9,46 \cdot 9$ & $43 \cdot 0$ & $40 \cdot 5,45 \cdot 6$ & $42 \cdot 4$ & $39 \cdot 9,44 \cdot 9$ & \\
\hline BMI category ${ }^{\dagger \dagger}$ & & & & & & & & & & & & & & & \\
\hline
\end{tabular}


well as compared with the rest of the labels in Canada, Mexico, the UK and the US (Table 2).

In Canada, Mexico and the US, HWL also led to lower perceived product healthfulness compared with the control group, but to a lesser magnitude than HIWL (range of $\beta$ : -0.50 to -0.33$)$. In Mexico, MTL also led to a decreased perceived healthfulness of the fruit drink compared with the control condition, with similar effects as the HWL $(\beta=-0 \cdot 26,95 \% \mathrm{CI}-0 \cdot 50,-0 \cdot 03)$.

In Australia, those in the HIWL and the HSR conditions had similar decreased perceptions of product healthfulness compared with the control group (range of $\beta:-0.81$ to $-0 \cdot 88$ ), as well as compared with the GDA, the MTL and the HWL (Table 2).

In the UK, all label conditions led to a decreased perceived healthfulness of the fruit drink compared with the control condition (Table 2). GDA, MTL and the HSR decreased the perceived product healthfulness to a similar extent in comparison with the control condition (range of $\beta$ : -0.31 to $-0 \cdot 46)$. HWL $(\beta=-0.50,95 \% \mathrm{CI}-0 \cdot 71,-0 \cdot 29)$ had a larger effect in decreasing perceived product healthfulness than GDA and the HSR.

In the UK, a statistically significant interaction between label condition and age group was observed (interaction effects $P<0.001$ ) (Fig. 3). This interaction indicated that the magnitude of the impact of HIWL compared with the control condition was greater among older age groups (i.e. $40-49$ years: $\beta=-1 \cdot 15,95 \% \mathrm{CI}-1 \cdot 64,-0 \cdot 66$; 50-59 years: $\beta=-1 \cdot 64,95 \% \mathrm{CI}-2 \cdot 04,-1 \cdot 23$; and 60 years and over: $\beta=-1 \cdot 71,95 \% \mathrm{CI}-2 \cdot 03,-1 \cdot 39)$ than it was among those aged $18-29$ years $(\beta=-0.63,95 \% \mathrm{CI}-1 \cdot 02,-0 \cdot 24)$ or $30-39$ years $(\beta=-0.45,95 \% \mathrm{CI}-0.92,0 \cdot 02)$.

No other differences in label effects across key demographic characteristics (i.e. gender, income adequacy, education, nutrition knowledge, food shopping in the household, frequency of using the nutrition facts table or BMI category) were observed within countries.

Sensitivity analyses suggested that there were few differences in key outcomes when comparing linear and logistic regression outcomes (online supplementary material, Supplemental Table 4).

\section{Discussion}

This study showed that the effect of FOP labels differed across countries. HIWL were the only FOP labels which consistently led participants to perceive the sweetened fruit drink as less healthy compared with the same drink without a label across all countries. In Australia only, there was a similar effect of HSR and HIWL. Other labels were effective in decreasing the perceived healthfulness of the drink within some countries only, but to a lower extent. The GDA did not exert this effect in most of the countries included in the study except the UK. In the UK, the effect of HIWL differed by age group, with greater impact among 
participants aged 40 and over. There were no other variations across key demographic characteristics in most countries, suggesting that different population sub-groups had similar responses to the various labelling systems.

These findings are consistent with a meta-analysis examining warning labels on sugary drinks, which showed that sugary drink warnings (HIWL or HWL) led to lower perceived product healthfulness compared with controls ${ }^{(25)}$. Similarly, recent studies comparing the effect of interpretive (e.g. HIWL, HSR and MTL) and reductive (i.e. GDA) FOP labelling schemes showed that interpretive labels had the greatest influence on product healthfulness perceptions ${ }^{(49)}$, with HIWL being the most effective among interpretive labels ${ }^{(50-52)}$. However, our findings are somewhat contrasting to reports by Ikonen et $a l^{(5)}$, a metaanalysis where increases in the perceived healthfulness of unhealthy products were observed for MTL and GDA, whereas no effect was reported for the HSR or HIWL. Differences may be explained by the types and relative healthfulness of products tested, and the amount of ambiguity related to their perceived healthfulness among consumers. Ikonen et al. included a variety of studies exploring the effects among different products, which were then re-classified as unhealthy or healthy products. However, studies suggest that larger impacts in perceived healthfulness are observed among products with intermediate healthfulness scores (e.g. breakfast cereals, yogurt, orange juice, bread), but less impact in products that people already believe are healthy (i.e. lentils and green beans) or unhealthy (i.e. potato chips) ${ }^{(49-52)}$. In our study, we used a sweetened fruit drink, which is often assumed to be a healthy option despite its high sugar content ${ }^{(34,35)}$. Given that only one type of food product was used in the experiment, one cannot assume the reported effect of labels will hold true for other food products, as has been demonstrated in other research ${ }^{(5)}$. Nonetheless, results build on evidence indicating that interpretive labelling schemes may be useful for decreasing perceived healthfulness of products with high content of nutrients associated with non-communicable diseases.

HIWL have become increasingly popular as a FOP label option to help consumers make healthier choices ${ }^{(53)}$. In contrast to most other labelling systems tested in this study, HIWL only highlight products with high amounts of critical nutrients (i.e. energy, fat, sugar and salt). Studies have shown that HIWL make excessive nutrient content and its negative health consequences more salient in consumers' minds ${ }^{(54)}$. Further, evidence indicates that the black colour and the octagon shape may have stronger implicit associations with unhealthfulness ${ }^{(55)}$. These characteristics may explain why HIWL may be more effective messaging to communicate the idea that a product is not healthful $^{(5,25,26,50,56,57)}$. We also observed that HIWL were more effective than HWL in communicating that the sweetened fruit drink was not healthy. Only a small number of studies have compared HIWL to HWL ${ }^{(58-62)}$, and more 


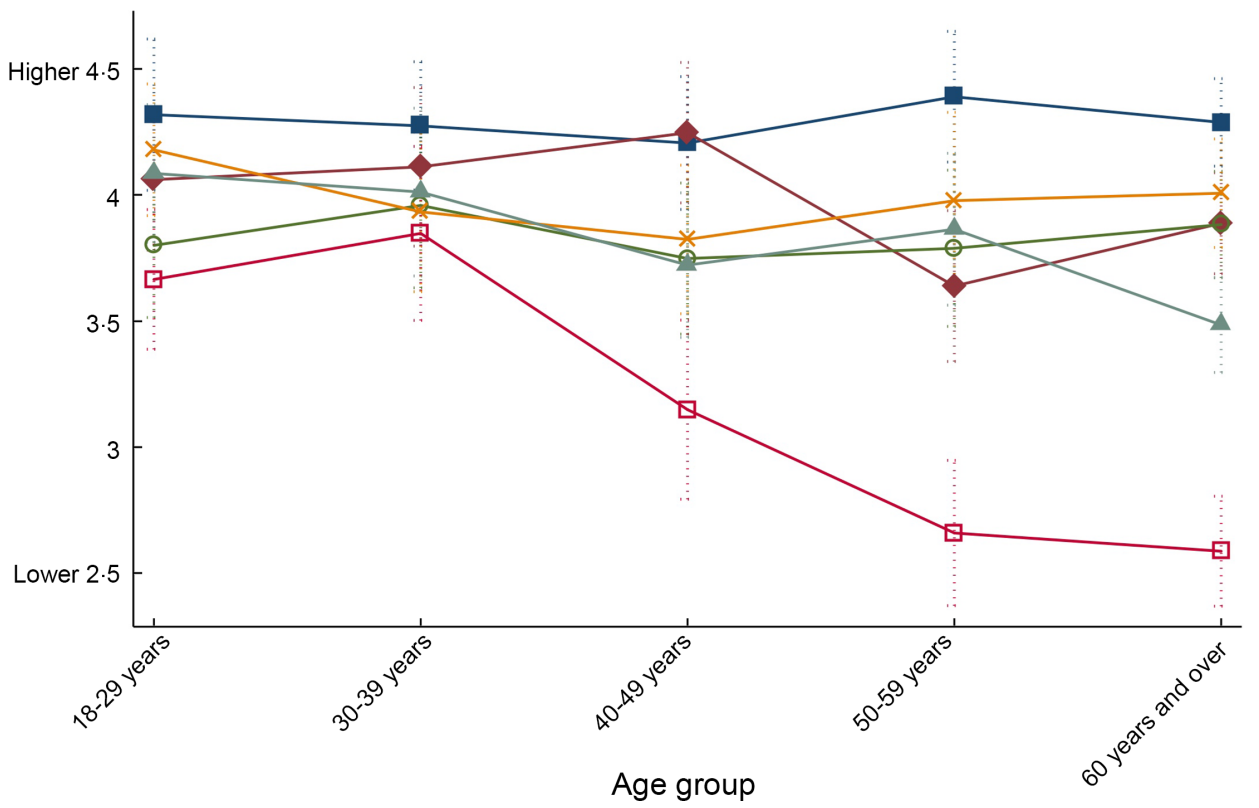

Fig. 3 Predicted perceived healthfulness by label condition across age groups in the UK. Predictions and $95 \% \mathrm{Cl}$ were estimated after running a linear regression model adjusted for the interaction term 'label condition $\times$ age group' and post-stratification sample weights. -- , Control; $\_$- Guideline Daily Amounts (GDA); $\_$, Multiple Traffic Lights (MTL); $\_$, Health Star Rating (HSR); $\_-$, Health Warning Labels (HWL); $\square$, 'High-in' Warning Labels (HIWL)

research is needed to continue answering important policy questions about how warnings can be most effectively used on food products. Future studies may also examine health warnings related to other unhealthy nutrients (e.g. Na) and for other less healthy product categories besides sugary drinks (e.g. processed meat).

In the current study, GDA had no effect on the perceived healthfulness of the product in most countries, except the UK. This finding is in line with evidence suggesting that reductive systems such as GDA, which rely on quantitative nutrient amounts, are not effective in communicating the presence of excessive amounts of critical nutrients in unhealthy foods ${ }^{(49)}$. As mentioned in previous reports ${ }^{(3)}$, these results suggest that interpretative FOP labelling systems, which incorporate elements of colour and symbolism and simplify information presented, hold more promise for conveying accurate information about product healthfulness to consumers.

Reports have also suggested that the effect of FOP labels may differ across countries. To date, most between-country studies exploring label perceptions (e.g. liking, understanding and use) or objective understanding of different FOP labels have been inconsistent, with some reporting differences across countries ${ }^{(27-29)}$, whereas others have $\operatorname{not}^{(30,31)}$. This study adds to the literature by investigating the effect of labels on perceived healthfulness of a fruit drink, finding several notable differences in the observed effect of labels across countries. It has been posited that familiarity with the labels (e.g. due to implementation of such labels and viewing labels on packages, or cultural exposure to public debates on issues of nutrition and labelling) may influence self-reported evaluations and usage intentions of labels ${ }^{(29,32)}$. In line with the former, the HSR was only effective in reducing perceptions of healthfulness in Australia where this policy is currently implemented on a voluntary basis; a similar effect was observed for MTL in the UK. In a broader sense, these results suggest that label effects may not be generalisable across countries and underscore the importance of producing local evidence to guide decision-making related to FOP nutrition labelling policies. Nonetheless, HIWL consistently led to lower perceived product healthfulness across all countries, suggesting that this format requires very little in the way of familiarity to be effective and may produce similar responses across high and upper-middle income countries.

The current study also examined whether the effect of labels differed across demographic characteristics. Overall, labels worked equally well across diverse populations. However, in the UK HIWL were more effective in decreasing perceived product healthfulness among older age groups than younger populations. Warning labels elicit a negative affect or perception of risk, which in turn may influence perceived product healthfulness ${ }^{(26)}$. Previous studies have reported greater health risk perceptions among adults and older adults compared with younger counterparts ${ }^{(63,64)}$, which may be explained by a greater exposure to health problems. However, the fact that label effects did not differ across income levels or nutrition knowledge, as shown in previous studies ${ }^{(65,66)}$, suggests that these labels are unlikely to contribute to increasing health disparities. 
To our knowledge, this is the first international study comparing the effect of different FOP labels on the perceived healthfulness of a food product among countries with varying government led or mandated FOP labelling policies implemented. This study also included one Latin American country, a region which has been previously understudied. Strengths of this study include the use of a randomised design, limiting the influence of confounding from observed and unobserved factors, and a large sample size. Nonetheless, results should be interpreted within the context of several limitations. Respondents were recruited using non-probability-based sampling; therefore, the findings do not provide nationally representative estimates. However, although the descriptive statistics may not match completely with national estimates of education and BMI, the observed effects in this study provide useful information regarding the potential effects of labels across a wider population. This study focused on examining the effect of labels in perceived healthfulness using a single item measure. To expand evidence on the effectiveness of labels to communicate the relative healthfulness of products, future studies should explore the effect of labels using multiple measures and across a range of healthy products, including direct comparisons between their healthfulness and likelihood of purchase. Further, the experiment was not performed in a store; therefore, the results might have been different among some participants in a real-life situation or shopping environment. However, online food shopping is becoming increasingly common in many countries and consumers are more accustomed to rating the healthfulness of a food product when shopping online. Results of this international labelling experiment provide relevant insights for policy- and decision-makers regarding FOP labelling systems.

\section{Conclusions}

Results indicate that warning labels are the most promising FOP labelling option to change consumer healthfulness perceptions. Specifically, HIWL may be particularly effective in helping consumers correctly identify unhealthy products with high contents of critical nutrients. Given that HIWL have been effectively implemented in several countries to date, and are compatible with international trade agreements, the current study adds to the evidence demonstrating that implementing HIWL on the front of packages is a strong policy option. The study supports the use of MTL in the UK, where this label has been implemented for more than 10 years, but has shown HIWL performed best in this country, especially among older age groups. Findings also support the consideration of the HSR for Australia, since this label performed better than the control and had a comparable effect to HIWL in this country, where this label has been implemented for more than 5 years. However, MTL were not effective outside the UK, and HSR was not effective outside Australia. Likewise, the study found little support for GDA as an option for a FOP labelling policy. Differences in label effects across countries highlight the importance of local evidence for guiding policy-making. Finally, different population sub-groups had similar responses to the various labelling systems tested in most countries, indicating FOP labels are unlikely to exacerbate disparities.

\section{Acknowledgements}

Acknowledgements: None. Financial support: The content is solely the responsibility of the authors and does not necessarily represent the official views of the funders. Funding for this project was provided by a Canadian Institutes of Health Research (CIHR) Project Grant, with additional support from the Public Health Agency of Canada (PHAC) and a CIHR-PHAC Applied Public Health Chair (DH). K01HL147713 from the National Heart, Lung, and Blood Institute of the National Institutes of Health supported Marissa Hall's time writing the paper. The study sponsor had no role in study design; collection, analysis and interpretation of data; writing the report; and the decision to submit the report for publication. Conflict of interest: There are no conflicts of interest. Authorship: A.J. performed the statistical analysis, interpreted the data and drafted the manuscript. C.M.W., L.V. and M.G.H. helped draft the manuscript, made substantial contributions in selecting the appropriate statistical analyses, interpreting the data and revising the manuscript critically for intellectual content. A.C.M., C.N., G.S., J.T. and S.B. made substantial contributions in interpreting the data, and revising the manuscript critically for intellectual content. J.T. provided statistical expertise and advice. A.J., C.M.W., L.V., G.S., J.T. and S.B. contributed to the preparation of the survey. D.H. conceived, designed and executed the International Food Policy Study. All authors revised the manuscript critically for intellectual content. All authors read and approved the final manuscript. Ethics of human subjectparticipation: This study was conducted according to the guidelines laid down in the Declaration of Helsinki. The study was reviewed by and received ethics clearance through a University of Waterloo Research Ethics Committee (ORE\# 30829). A full description of the study methods can be found in the International Food Policy Study: Technical Report - Wave 2 (2018) at www.foodpolicystudy.com/ methods. Written informed consent was obtained from all participants.

\section{Supplementary material}

For supplementary material accompanying this paper visit https://doi.org/10.1017/S1368980021004535 


\section{References}

1. Branca F, Lartey A, Oenema S et al. (2019) Transforming the food system to fight non-communicable diseases. BMJ 364, 1296.

2. McGuire S (2016) Scientific report of the 2015 dietary guidelines advisory committee. Washington, DC: US Departments of Agriculture and Health and Human Services, 2015. Adv Nutr 7, 202.

3. Wartella EA, Lichtenstein AH, Yaktine A et al. (2012) Frontof-Package Nutrition Rating Systems and Symbols: Promoting Healthier Choices. Washington, DC: The National Academies Press, Institute of Medicine.

4. FAO/WHO (2016). Joint FAO/WHO Food Standards Programme Codex Committee on Food Labelling. Proceedings of the Forty-Third Session. Proposal for New Work Concerning A Global Standard for Front Of Pack Interpretive Nutrition Labelling. https://www.fao.org/faowho-codexalimentarius/sh-proxy/es/?lnk=1\&url=https:// workspace.fao.org/sites/codex/Meetings/CX-714-43/CRD/fl43_ CRD20x.pdf (accessed November 2020).

5. Ikonen I, Sotgiu F, Aydinli A et al. (2019) Consumer effects of front-of-package nutrition labeling: an interdisciplinary meta-analysis. J Acad Mark Sci 48, 360-383.

6. Kanter R, Vanderlee L \& Vandevijvere S (2018) Front-ofpackage nutrition labelling policy: global progress and future directions. Public Health Nutr 21, 1399-1408.

7. Morrison N (2016) UK Traffic Light Labelling Should Be Mandatory: LGA. Food Navigator. https://www.foodnavi gator.com/Article/2016/09/20/UK-traffic-light-labelling-sho uld-be-mandatory-LGA (accessed November 2021).

8. Ecuador \& Ministerio de Salud Pública (2013) Reglamento de Etiquetado de Alimentos Procesados Para el Consumo Humano. Acuerdo No. 00004522. https://www.controlsanit ario.gob.ec/wp-content/uploads/downloads/2014/08/REGLA MENTO-SANITARIO-DE-ETIQUETADO-DE-ALIMENTOS-PR OCESADOS-PARA-EL-CONSUMO-HUMANO-junio-2014. pdf (accesed November 2021).

9. The Gazette of the Democratic Socialist Republic of Sri Lanka (2019) Food (Colour Coding for Sugar, Salt and Fat) Regulations 2019 - No 26/1980. http://eohfs.health.gov.lk/ food/images/pdf/regulations/Colour-coding-solids-English. pdf (accessed February 2021).

10. Zargaraan A, Dinarvand R \& Hosseini H (2017) Nutritional traffic light labeling and taxation on unhealthy food products in Iran: health policies to prevent non-communicable diseases. Iran Red Crescent Med J 19, e 57874.

11. Australian Government Department of Health (2015) Guide for Industry to the Health Star Rating Calculator (HSRC). Canberra: Australian Government Department of Health.

12. Jones A, Shahid M \& Neal B (2018) Uptake of Australia's health star rating system. Nutrients 10, 997.

13. Pan American Health Organization (2020) Front-of-Package Labeling as a Policy Tool for the Prevention of NonCommunicable Diseases in the Americas. Washington, DC: Pan American Health Organization.

14. Diario Oficial (2020) MODIFICACIÓN a la Norma Oficial Mexicana NOM-051-SCFI/SSA1-2010, Especificaciones Generales de Etiquetado Para Alimentos y Bebidas no Alcohólicas Preenvasados-Información Comercial y Sanitaria, Publicada el 5 de Abril de 2010. https://www.dof. gob.mx/2020/SEECO/NOM_051.pdf (accessed November 2019).

15. Diário Oficial da UNIÃO (2020) Resolução de Diretoria Colegiada - RDC no 429, de 8 de Outubro de 2020. https:// www.in.gov.br/en/web/dou/-/resolucao-de-diretoria-colegia da-rdc-n-429-de-8-de-outubro-de-2020-282070599 (accessed November 2021).

16. Argentina D (2019) Etiquetado Frontal Informativo de Alimentos o Sustancias Aptas Para Consumo Humano.
Regimen. https://www.diputados.gov.ar/proyectos/proye ctoTP.jsp?exp=4995-D-2019 (accessed January 2020).

17. Canada Gazzette, Food and Drugs Act. (2018) Regulations Amending Certain Regulations Made Under the Food and Drugs Act (Nutrition Symbols, Other Labelling Provisions, Partially Hydrogenated Oils and Vitamin D). https:// gazette.gc.ca/rp-pr/p1/2018/2018-02-10/html/reg2-eng.html (accessed November 2021).

18. Pomeranz JL, Mozaffarian D \& Micha R (2020) Sugar-sweetened beverage warning policies in the broader legal context: health and safety warning laws and the first amendment. $\mathrm{Am}$ J Prev Med 58, 783-788.

19. Roberto CA, Ng SW, Ganderats Fuentes M et al. (2021) The influence of front-of-package nutrition labeling on consumer behavior and product reformulation. Annu Rev Nutr 41, 529-550.

20. Burton S, Tangari AH, Howlett E et al. (2014) How the perceived healthfulness of restaurant menu items influences sodium and calorie misperceptions: implications for nutrition disclosures in chain restaurants. $J$ Consum Aff 48, 62-95.

21. Sogari G, Li J, Lefebvre M et al. (2019) The influence of health messages in nudging consumption of whole grain pasta. Nutr 11, 2993.

22. Franco-Arellano B, Vanderlee L, Ahmed M et al. (2020) Influence of front-of-pack labelling and regulated nutrition claims on consumers' perceptions of product healthfulness and purchase intentions: a randomized controlled trial. Appetite 149, 104629.

23. Borgmeier I \& Westenhoefer J (2009) Impact of different food label formats on healthiness evaluation and food choice of consumers: a randomized-controlled study. BMC Public Health 9, 184 .

24. Khandpur N, de Morais Sato P, Mais LA et al. (2018) Are front-of-package warning labels more effective at communicating nutrition information than traffic-light labels? A randomized controlled experiment in a Brazilian sample. Nutrients 10, 1-15.

25. Grummon AH \& Hall MG (2020) Effectiveness of sugary drink warnings: a meta-analysis of experimental studies. PLoS One 17, e1003120.

26. Taillie LS, Hall MG, Popkin BM et al. (2020) Experimental studies of front-of-package nutrient warning labels on sugar-sweetened beverages and ultra-processed foods: a scoping review. Nutrients 12, 569.

27. Möser A, Hoefkens C, van Camp J et al. (2010) Simplified nutrient labelling: consumers' perceptions in Germany and Belgium. J Verbr Lebensm 5, 169-180.

28. Egnell M, Talati Z, Hercberg S et al. (2018) Objective understanding of front-of-package nutrition labels: an international comparative experimental study across 12 countries. Nutrients 10, 1542.

29. Grunert KG, Fernández-Celemín L, Wills JM et al. (2010) Use and understanding of nutrition information on food labels in six European countries. J Public Health 18, 261-277.

30. Talati Z, Egnell M, Hercberg S et al. (2019) Consumers' perceptions of five front-of-package nutrition labels: an experimental study across 12 countries. Nutrients 11, 1934.

31. Feunekes GIJ, Gortemaker IA, Willems AA et al. (2008) Front-of-pack nutrition labelling: testing effectiveness of different nutrition labelling formats front-of-pack in four European countries. Appetite 50, 57-70.

32. Van Herpen E, Seiss E \& Van Trijp HCM (2012) The role of familiarity in front-of-pack label evaluation and use: a comparison between the United Kingdom and The Netherlands. Food Qual Prefer 26, 22-34.

33. Talati Z, Norman R, Pettigrew S et al. (2017) The impact of interpretive and reductive front-of-pack labels on food choice and willingness to pay. Int J Behav Nutr Phys Act 14, 1-10. 
34. Moran AJ \& Roberto CA (2018) Health warning labels correct parents' misperceptions about sugary drink options. Am J Prev Med 55, e19-e27.

35. Auerbach BJ, Dibey S, Vallila-Buchman P et al. (2018) Review of $100 \%$ fruit juice and chronic health conditions: implications for sugar-sweetened beverage policy. Adv Nutr 9, 78-85.

36. Popkin BM \& Hawkes C (2016) Sweetening of the global diet, particularly beverages: patterns, trends, and policy responses. Lancet Diabetes Endocrinol 4, 174-186.

37. UK Department of Health (2016) Guide to Creating a Front of Pack (FoP) Nutrition Label for Pre-Packaged Products Sold Through Retail Outlets. https://www.food.gov.uk/sites/ default/files/media/document/fop-guidance_0.pdf (accessed March 2020).

38. Commonwealth of Australia (2020) Health Star Rating Calculator and Artwork. http://www.healthstarrating.gov.au/ internet/healthstarrating/publishing.nsf/Content/calculator (accessed October 2021).

39. Corvalán C, Reyes M, Garmendia ML et al. (2019) Structural responses to the obesity and non-communicable diseases epidemic: update on the Chilean law of food labelling and advertising. Obes Rev 20, 367-374.

40. Ministerio de Salud \& Gobierno de Chile (2017) Manual de Etiquetado Nutricional de Alimentos. https://www.minsal. cl/wp-content/uploads/2018/01/Manual-Etiquetado-Nutrici onal-Ed.-Minsal-2017v2.pdf (accesed November 2021).

41. Hammond D, White CM \& Mahamad S (2018) International Food Policy Study: Methods. Waterloo: University of Waterloo.

42. Centers for Disease Control and Prevention (2016) Behavioral Risk Factor Surveillance System. https://www. cdc.gov/brfss/index.html (accessed November 2021)

43. Centers for Disease Control and Prevention (2016) National Health and Nutrition Examination Survey. https://www.cdc. gov/nchs/nhanes/index.htm (accessed November 2021).

44. Statistics Canada (2013) Canadian Community Health Survey Annual Component (CCHS). https://www.statcan.gc.ca/en/ survey/household/3226 (accessed November 2021).

45. Australia Bureau of Statistics (2016) The 2016 Census of Population and Housing. https://www.abs.gov.au/ausstats/ abs@.nsf/lookup/by subject/1001.0 2016-17 main featur es the 2016 census of population and housing 10009 (accessed November 2021).

46. Institito Nacional de Estadística y Geografía (2010) Censo de Población y Vivienda 2010. Consulta Interactiva de Datos. https://www.inegi.org.mx/programas/ccpv/2010/ default.html?init=1 (accesed November 2021).

47. Litwin H \& Sapir EV (2009) Perceived income adequacy among older adults in 12 countries: findings from the survey of health, ageing, and retirement in Europe. Gerontologist 49 , 397-406.

48. Hammond D, White CM, Rynard VL et al. (2019) International Food Policy Study: Technical Report - 2018 Survey (Wave 2). Waterloo: University of Waterloo.

49. Arrúa A, MacHín L, Curutchet MR et al. (2017) Warnings as a directive front-of-pack nutrition labelling scheme: comparison with the guideline daily amount and traffic-light systems. Public Health Nutr 20, 2308-2317.

50. Lima M, Ares G \& Deliza R (2018) How do front of pack nutrition labels affect healthfulness perception of foods targeted at children? Insights from Brazilian children and parents. Food Qual Prefer 64, 111-169.

51. Ares G, Varela F, Machin L et al. (2018) Comparative performance of three interpretative front-of-pack nutrition labelling schemes: insights for policy making. Food Qual Prefer $\mathbf{6 8}$, 215-225.

52. Acton RB, Vanderlee L \& Hammond D (2018) Influence of front-of-package nutrition labels on beverage healthiness perceptions: results from a randomized experiment. Prev Med 115, 83-89.

53. Pan American Health Organization (2020) Front-of-Package Labeling as a Policy Tool for the Prevention of NonCommunicable Diseases in the Americas. Washington D.C.: Pan American Health Organization.

54. Ares G, Antúnez L, Otterbring T et al. (2020) Sick, salient and full of salt, sugar and fat: understanding the impact of nutritional warnings on consumers' associations through the salience bias. Food Qual Prefer 86, 103991.

55. Cabrera M, Machín L, Arrúa A et al. (2017) Nutrition warnings as front-of-pack labels: influence of design features on healthfulness perception and attentional capture. Public Health Nutr 20, 3360-3371.

56. Roberto CA, Wong D, Musicus A et al. (2016) The influence of sugar-sweetened beverage health warning labels on parents' choices. Pediatrics 137, e20153185.

57. Vanderlee L, Franco-Arellano B, Ahmed M et al. (2021) The efficacy of 'high in' warning labels, health star and traffic light front-of-package labelling: an online randomised control trial. Public Health Nutr 24, 62-74.

58. Ang FJL, Agrawal S \& Finkelstein EA (2019) Pilot randomized controlled trial testing the influence of front-of-pack sugar warning labels on food demand. BMC Public Health 19, 164.

59. Acton RB \& Hammond D (2018) The impact of price and nutrition labelling on sugary drink purchases: results from an experimental marketplace study. Appetite 121, 129-137.

60. Hall MG, Lazard AJ, Grummon AH et al. (2021) Designing Impactful Warnings for Sugary Drinks: A Randomized Clinical Trial with Latino and Non-Latino Parents. Prev Med 148, 106562

61. Grummon AH, Hall MG, Taillie LS et al. (2019) How should sugar-sweetened beverage health warnings be designed? A randomized experiment. Prev Med 121, 158-166.

62. Bollard T, Maubach N, Walker N et al. (2016) Effects of plain packaging, warning labels, and taxes on young people's predicted sugar-sweetened beverage preferences: an experimental study. Int J Behav Nutr Phys Act 13, 95.

63. Kim Y, Park I \& Kang S (2018) age and gender differences in health risk perception. Cent Eur J Public Health 26, $54-59$.

64. Cohn LD, Macfarlane S, Yanez C et al. (1995) Risk-perception: differences between adolescents and adults. Health Psychol 14, 217-222.

65. Grummon AH, Taillie LS, Golden SD et al. (2019) Sugarsweetened beverage health warnings and purchases: a randomized controlled trial. Am J Prev Med 57, 601-610.

66. Grummon AH, Smith NR, Golden SD et al. (2019) Health warnings on sugar-sweetened beverages: simulation of impacts on diet and obesity among U.S. adults. Am J Prev Med 57, 765-774. 